\title{
Pharmacogenomics of paclitaxel
}

The microtubule-stabilizing drug paclitaxel is a cytotoxic agent widely used for the treatment of a variety of tumor types. Since its introduction to the clinic, modifications to the administration schedule and treatments for hypersensitivity reactions and neutropenia have significantly improved paclitaxel therapy. On the other hand, severe neurotoxicity and lack of response are still clinical challenges. During the last decade a deeper knowledge of paclitaxel pharmacokinetics and pharmacodynamics has been achieved, together with an in-depth characterization of genes involved in its elimination and therapeutic response. Pharmacogenetic studies aimed at the identification of paclitaxel outcome biomarkers have been performed, however, further efforts will be required to successfully integrate these and future results to provide the basis for personalized paclitaxel therapy.

\section{KEYWORDS: efficacy neurotoxicity paclitaxel pharmacogenetics polymorphisms Taxol $^{\circledR}$}

\section{Past}

Paclitaxel is a natural alkaloid isolated from the bark of a tree, Taxus brevifolia, currently used to treat a variety of tumors, including ovarian, breast and non-small-cell lung cancers. Paclitaxel anti-tumoral activity was discovered in the 1970s and it was first approved by the US FDA in 1992. The mechanism of action was established as the stabilization of cellular microtubules and blocking of chromosome segregation [1], and in the years following, the $\beta$-tubulin functional domains and the domains containing paclitaxel-binding sites were identified [2].

The administration of paclitaxel $\left(\operatorname{Taxol}^{\circledR}\right)$ through intravenous infusions was achieved by using Cremophor ${ }^{\circledR} \mathrm{EL}$ as a vehicle to entrap the drug in micelles and keep it in solution, which affects the disposition of paclitaxel and is responsible for the nonlinear pharmacokinetics of the drug, especially at higher dose levels. Hepatic metabolism and biliary excretion were determined as the primary routes of systemic paclitaxel elimination [3], and paclitaxel metabolites were identified as $6 \alpha$-hydroxypaclitaxel, p-hydroxy-C3'-paclitaxel and dihydroxypaclitaxel. With the use of inhibitors and in vitro models, cytochrome P450 2C8 (CYP2C8) and CYP3A $4 / 5$ were discovered to form these metabolites, and P-glycoprotein was found to mediate paclitaxel efflux.
The major adverse effects encountered with Taxol were severe hypersensitivity reactions, mainly attributed to Cremophor EL; hematologic toxicity, primarily appearing in the form of severe neutropenia; and neurotoxicity, mainly seen as cumulative sensory peripheral neuropathy. Paclitaxel safety was greatly improved by diminishing the hypersensitivity reactions with corticosteroids and $\mathrm{H} 1$ and $\mathrm{H} 2$ antihistamine premedication, and by reducing the incidence of grade $3 / 4$ neutropenia with the administration of granulocyte colony-stimulating factors and shortening paclitaxel infusion time from 24 to $3 \mathrm{~h}$. On the other hand, the neurotoxicity, which was believed to be caused by either paclitaxel or Cremophor EL, could not be controlled and became the dose-limiting toxicity of the drug.

With respect to efficacy, paclitaxel was found to be active for several tumor types and its use was extended from ovarian to breast and lung cancers. To predict response, different mechanisms were investigated. P-glycoprotein overexpression was shown to confer resistance to paclitaxel, although demonstrating its role in vivo has been problematic and remains a point of conflict [4]. In non-small-cell lung cancer, mutations in the therapeutic target, $\beta$-tubulin, were suggested to be strong predictors of response [5]. However, subsequent studies avoiding cross reactions with pseudogenes could not confirm this finding, and

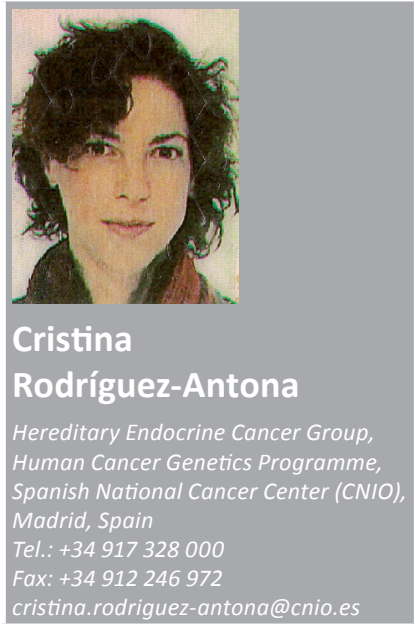


to date, mutations in $\beta$-tubulin genes have only been shown to confer resistance to paclitaxel in cell lines in vitro.

\section{Present}

In the last decade, our knowledge of paclitaxel clinical pharmacology has significantly improved, and important efforts have been made to identify genetic and nongenetic factors responsible for the interindividual variability of paclitaxel toxicity and efficacy. More sensitive methods to measure the concentrations of paclitaxel and its metabolites in plasma, as well as methods to determine the concentration of unbound paclitaxel (drug not bound to Cremophor EL or plasma proteins), which is the pharmacologically active form of the drug, were developed, together with improved models to predict paclitaxel pharmacokinetics-pharmacodynamics, such as the duration of plasma concentration above $0.05 \mu \mathrm{M}$ for paclitaxel [6]. In regards to neurotoxicity, pharmacokinetic studies $[7,8]$ and alternative paclitaxel formulations indicated that paclitaxel rather than Cremophor EL was the most important compound responsible for this adverse effect. The mechanism for the neurotoxicity has been demonstrated to involve ganglioneuropathy and axonopathy caused by dysfunctional microtubules in dorsal root ganglia, axons and Schwann cells, and efforts have been made to try to identify strategies to manage this toxicity (e.g., by using neuroprotective agents), although these attempts have yielded little success in the clinical setting. Paclitaxel dose, infusion duration, schedule of administration, prior exposure to neurotoxic agents and pre-existing medical disorders were identified as neurotoxicity risk factors. However, a large extent of the interindividual variability in neurotoxicity remains unexplained.

Variability in paclitaxel pharmacokinetics has been associated with the adverse effects of the drug. Thus, polymorphisms in genes encoding paclitaxel-metabolizing enzymes, transporters and therapeutic targets have been suggested to contribute to the interindividual variability in toxicity and response. In this respect, during the past decade, further characterization of genes involved in paclitaxel elimination and drug response was performed, including the identification of their most relevant genetic variants. The organic anion transporting polypeptide (OATP) 1B3 was identified as a key protein for paclitaxel hepatic uptake [9], and polymorphisms in the genes encoding for paclitaxel metabolizing enzymes and transporters (CYP2C8, CYP3A4,
CYP3A5, P-glycoprotein and OATP1B3) have been described. It is important to note that the allele frequencies for many of these polymorphisms are subject to important ethnicity specific differences, with some alleles exclusively present in specific populations (e.g., the Caucasian $C Y P 2 C 8^{*} 3$ ), and that linkage disequilibrium among the polymorphisms in these genes can differ among populations (e.g., ATP-binding casette subfamily B member 1 [ $A B C B 1]$, which encodes P-glycoprotein), thereby hampering studies with tag-SNPs that are in linkage disequilibrium with functional variants. For the CYP2C8 gene, two alleles common in Caucasians that result in amino acid changes $C Y P 2 C 8^{*} 3$ (R139K; K399R) and CYP2C8*4 (I264M), were described. The former has been shown to possess an altered activity, while the latter does not seem to have functional consequences [10]. In addition, two CYP2C8 haplotypes were recently shown to confer an increased and reduced metabolizing activity, respectively [11]. CYP3A4 was proven to be well conserved with no common functional polymorphisms, while $C Y P 3 A 5$ was found to be highly polymorphic owing to $C Y P 3 A 5^{*} 3, C Y P 3 A 5^{*} 6$ and $C Y P 3 A 5^{*} 7$ [12], with the latter two being African-specific polymorphisms. For the uptake transporter OATP1B3, missense polymorphisms with functional consequences have been reported [13] and for P-glycoprotein three polymorphisms (1236C > T, 2677G $>\mathrm{T}$ and 3435C $>\mathrm{T}$ ) have been extensively studied, producing different results [14].

Pharmacogenetic studies comparing the most relevant polymorphisms in these genes and paclitaxel pharmacokinetics have rendered contradictory results, with some studies finding no associations while others reported an effect for $A B C B 1$, $C Y P 3 A 4$ or $C Y P 2 C 8$ polymorphisms on specific pharmacokinetic parameters. Again, with respect to paclitaxel neurotoxicity risk, some studies have rendered positive results for $A B C B 1[8,15]$, $C Y P 2 C 8$ [8,16] and CYP3A5 [16] polymorphisms, while others found no significant associations [17]. These differences might be caused by underpowered studies and by differences in the patients under study. With respect to the interindividual variations in paclitaxel efficacy and germline polymorphisms, conflicting results have been found. However, changes affecting microtubule structure and/or composition have been shown to affect paclitaxel efficacy, probably by reducing drug-target affinity. Mainly, resistance to tubulin-binding agents has been associated with an overexpression of $\beta$-tubulin isotype III [18], which seems to be caused by a deregulation of the microRNA family 200 [LESKELÄ S ET AL. MANUSCRIPT 
submitted] [19]. However, the clinical utility of these findings remains to be established; furthermore, the identification of biomarkers that could be used to individualize paclitaxel treatment remains a challenge.

\section{Future}

Despite the recent advancements aimed at optimizing paclitaxel therapy, important clinical challenges remain unresolved, such as the inability to prevent, treat, or predict paclitaxel neurotoxicity, and lack of biomarkers capable of predicting response. Thus, in the coming years, further efforts will be required to provide an individualized paclitaxel pharmacotherapy. To achieve this, both therapeutic drug monitoring and genetic testing for outcome markers might be relevant, taking into account that polymorphisms associated with neurotoxicity will most likely be valid for patients with different tumors, but that different efficacy markers might need to be identified and optimized for each cancer type. Importantly, this identification of genes associated with paclitaxel outcome might also provide the basis for new therapeutic strategies aimed at decreasing

\section{Bibliography}

1 Manfredi JJ, Horwitz SB: Vinblastine paracrystals from cultured cells are calciumstable. Exp. Cell Res. 150, 205-212 (1984).

2 Nogales E, Wolf SG, Downing KH: Structure of the $\alpha \beta$ tubulin dimer by electron crystallography. Nature 391, 199-203 (1998).

3 Rowinsky EK, Wright M, Monsarrat B, Donehower RC: Clinical pharmacology and metabolism of taxol (paclitaxel): update 1993. Ann. Oncol. 5(Suppl. 6), S7-S16 (1994).

4 Pusztai L: Markers predicting clinical benefit in breast cancer from microtubule-targeting agents. Ann. Oncol. 18(Suppl. 12), xii15-20 (2007).

5 Monzo M, Rosell R, Sanchez JJ et al.: Paclitaxel resistance in non-small-cell lung cancer associated with $\beta$-tubulin gene mutations. J. Clin. Oncol. 17, 1786-1793 (1999).

6 Joerger M, Huitema AD, Richel DJ et al.: Population pharmacokinetics and pharmacodynamics of paclitaxel and carboplatin in ovarian cancer patients: a study by the European Organization for Research and Treatment of Cancer-Pharmacology and Molecular Mechanisms Group and New Drug Development Group. Clin. Cancer Res. 13, 6410-6418 (2007).

7 Mielke S, Sparreboom A, Steinberg SM et al.: Association of paclitaxel pharmacokinetics with the development of

toxicity without compromising drug efficacy. To successfully carry out this work, national and international pharmacogenomics consortia will be required to launch large collaborative studies with adequate numbers of homogenous and wellcharacterized samples. Candidate gene approaches have been successful for many pharmacogenetic studies. However, genome-wide approaches will also be required to facilitate the identification of genes still unconnected with paclitaxel but important for outcome. Ultimately, prospective pharmacogenetic-guided clinical trials will be required in order to accurately establish the utility of the identified markers/strategies for patients and healthcare systems.

\section{Financial \& competing interests disclosure}

This article was supported by the Spanish Ministry of Science and Technology (SAF2009-08307). The author has no other relevant affiliations or financial involvement with any organization or entity with a financial interest in or financial conflict with the subject matter or materials discussed in the manuscript apart from those disclosed.

No writing assistance was utilized in the production of this manuscript.

peripheral neuropathy in patients with advanced cancer. Clin. Cancer Res. 11, 4843-4850 (2005).

8 Green H, Soderkvist P, Rosenberg P et al: Pharmacogenetic studies of paclitaxel in the treatment of ovarian cancer. Basic Clin. Pharmacol. Toxicol. 104, 130-137 (2009).

9 Smith NF, Acharya MR, Desai N, Figg WD, Sparreboom A: Identification of OATP1B3 as a high-affinity hepatocellular transporter of paclitaxel. Cancer Biol. Ther. 4, 815-818 (2005).

10 Niemi M, Leathart JB, Neuvonen M, Backman JT, Daly AK, Neuvonen PJ: Polymorphism in CYP2C8 is associated with reduced plasma concentrations of repaglinide. Clin. Pharmacol. Ther. 74, 380-387 (2003).

11 Rodriguez-Antona C, Niemi M, Backman JT et al:: Characterization of novel CYP2C8 haplotypes and their contribution to paclitaxel and repaglinide metabolism. Pharmacogenomics J. 8, 268-277 (2008).

12 Kuehl P, Zhang J, Lin Y et al.: Sequence diversity in $C Y P 3 A$ promoters and characterization of the genetic basis of polymorphic CYP3A5 expression. Nat. Genet. 27, 383-391 (2001).

13 Smith NF, Marsh S, Scott-Horton TJ et al.: Variants in the SLCO1B3 gene: interethnic distribution and association with paclitaxel pharmacokinetics. Clin. Pharmacol. Ther. 81, 76-82 (2007).
14 Leschziner GD, Andrew T, Pirmohamed M, Johnson MR: $A B C B 1$ genotype and Pgp expression, function and therapeutic drug response: a critical review and recommendations for future research. Pharmacogenomics J. 7, 154-179 (2007).

15 Sissung TM, Mross K, Steinberg SM et al.: Association of $A B C B 1$ genotypes with paclitaxel-mediated peripheral neuropathy and neutropenia. Eur. J. Cancer 42, 2893-2896 (2006).

16 Leskelä S, Jara C, Leandro-García LJ et al.: Polymorphisms in cytochromes P450 2C8 and $3 \mathrm{~A} 5$ are associated with paclitaxel neurotoxicity. Pharmacogenomics J. (2010) (Epub ahead of print).

17 Marsh S, Paul J, King CR, Gifford G, McLeod HL, Brown R: Pharmacogenetic assessment of toxicity and outcome after platinum plus taxane chemotherapy in ovarian cancer: the Scottish randomised trial in ovarian cancer. J. Clin. Oncol. 25 , 4528-4535 (2007).

18 Seve P, Dumontet C: Is class III $\beta$-tubulin a predictive factor in patients receiving tubulin-binding agents? Lancet Oncol. 9, 168-175 (2008).

19 Cochrane DR, Spoelstra NS, Howe EN, Nordeen SK, Richer JK: MicroRNA-200c mitigates invasiveness and restores sensitivity to microtubule-targeting chemotherapeutic agents. Mol. Cancer Ther. (2009) (Epub ahead of print). 